\title{
Energy Efficient Blood Transfusion System
}

\author{
Abhishek Rathi ${ }^{1}$, Gitesh Das $^{2}$, and Monica Subashini $\mathrm{M}^{2 *}$ \\ ${ }^{1}$ School of Information Technology and Engineering, Vellore Institute of Technology, Vellore \\ ${ }^{2}$ School of Electrical Engineering, Vellore Institute of Technology, Vellore
}

\begin{abstract}
RBCs stand for red blood cells (also known as erythrocytes), are the most common type of blood cells. They are the basic means of delivering oxygen, in the vertebrates to different cells and tissues. Hemoglobin is a protein in the RBCs, which carries oxygen and gives blood its red color. The normal range of RBCs in men is approximately 4.7 to 6.1 million cells per microliter and the normal range of RBCs in women is approximately 4.2 to 5.4 million cells per microliter. For a person to be eligible to donate blood, he/she should have the minimum number of RBCs in their blood for donation. This is only possible when the minimum duration between two donations is 3 months, be- cause it takes RBC about three months to develop completely. Many equipment have been developed or installed in hospitals for reducing energy consumption and increasing the efficiency. But even after installing these energy efficient devices in hospitals, it has been found out that these devices face a number of difficulties or obstacles. Thus, this paper focuses in developing an energy efficient software for blood transfusion that can function without interfering with any obstacles and thus giving accurate results.
\end{abstract}

\section{Introduction}

Blood transfusion has become common nowadays and thus it is important to check if the patient is getting the blood unit that has been assigned to them. Thereby, it is equally important to check the status of the person who is donating blood, i.e, the health check-up of the person donating blood. Among these, the important thing to check about the donor is that if the minimum duration gap between the two donations is three months or not.

\section{Literature survey}

[1]An Efficient Inventory Model to reduce the wastage of blood in the National Blood Trans- fusion Service: The researchers have mainly focused on developing an efficient blood inventory management model to reduce the platelet shortages. It has been seen that blood banks keep inventory levels according to their instincts due to which in the case of emergency, number of platelets required is not available which puts the patient's life at risk. Therefore, this paper focuses on how we can overcome these problems. [2] Using Simulation to Improve the Blood Supply Chain: It is very costly and difficult to get platelets from another blood bank in a short notice.

\footnotetext{
*Corresponding Author: monicasubashini.m@gmail.com
} 
Therefore, this paper focuses on how we can overcome these problems by improving the procedures and results or outcomes by modelling the entire blood supply chain. So, they have developed three algorithms (or three phases) based on certain assumptions: Blood banks are taking all the blood that is donated by donors; all the inventory levels are updated to the remaining shelf life on a daily basis; in these models, the platelets are issued to the wards based on first in - first out rule; the inventory levels are checked before taking blood from donors; in case if the desired level of blood inventory is not met, and if the donors are also not available then orders are placed from other blood banks to get platelets. The area is fruitful for future research like developing algorithms for scheduling donors or clustering the blood-banks island-wide.[3] An electronic barrier system to improve blood transfusion safety: In this paper, the authors have mainly focused on the implementation of an electronic based physical barrier (micro-controller based barrier system integrated with common barcode/RFID-based solutions). Whenever, in case of blood transfusion, it is important to check whether the patient is getting the blood unit that has been assigned to him or not. To ensure that the patients get the assigned blood unit, barcode labels and RFID tags have been used for the past few years.[4] Sources of Preventable Errors Related to Transfusion: Transfusion errors are not often reported because of lack of awareness or information about transfusion related events among the hospital staff and the centers. More effective and reliable results have been obtained when a barrier is used, that is mechanical devices that prevent the use of blood units until the cross-checking is done. Thus, this paper focuses on implementing a micro-controller based barrier system. This system provides blood unit temperature monitoring system with event-logging capabilities, and can be controlled wirelessly, powered by high capacity Li-ion battery, which ensures high operating cycles. [5] Improvement in Transfusion Safety: They have focused on improving the safety of the whole transfusion chain, technological solutions addressing the speed and reliability of standard cross-checking methods. Thus, the safety of the whole transfusion process is increased and the patient is given the assigned blood unit. [6] Safety Concerns in Blood Donations: Blood donation is a risky process and the amount of health issues it possess is lethal. Though some hospitals collect blood directly from the volunteers, the overall process of blood transfusion is risky. Some of the donors can have reactions and these reactions can lead to a serious injury. Thus, they have focused on improving the safety of the whole transfusion process. [7] Blood Donor's Safety using Data Mining: The health department has invented a software tool named "DONORHART" to collect and analyze reactions that occur at different blood centers. Data mining is used to analyze the factors influencing the blood donation process and provide insights on how can the transfusion system be improved and made less dangerous. This paper provides two cases performed on data reported to DonorHART tool:-A) Multiple vasovagal reactions B) Delayed vasovagal reactions with loss of consciousness. The donors can expect vasovagal reactions after completion of the donation and these delayed reactions can occur anywhere ranging from the place where they donated blood or off the blood donation site. Delayed vasovagal reactions (VVR) can lead to injuries and can prove to be fatal. Nonetheless blood donation is a very safe process and is taken care of by the authorities. Females have a higher occurrence of delayed VVR whereas Asians and Africans are also prone to VVR [8] Electronic Blood Transfusion System: Hospitals have successfully incorporated electronic blood transfusion systems. This has led to several positive consequences. The blood donation quality has been improved significantly and also the time taken to donate blood. Blood wastage involved in normal blood transfusion system has been taken into consideration and the staff hired for the process has also been reduced. The system has been made fully automatized. The proposal is to re-engineer the hospital services for blood transfusion: Barcode systems can be used to identify patients and digital computers can be used to prompt the staff members 
on every step involved in blood transfusion. This system uses 2 bar codes. One is with the patient and one is with the system. By identifying the patient using the system barcode, the correct setup is created. Usage of an automated blood transfusion system can deliver the results at a fast rate and benefit the patient being offered treatment. The process involves linking the transfusion lab to other IT systems, provides robust documentation and data transfer of information relevant to the transfusion practice at all stages of the transfusion process. [9] Development of an Automatic Electronic System to Human Blood Typing: This paper focuses on developing a low cost, lower maintenance, portable and automatic electronic system. Why use electronic blood transfusion system? Overcoming the poor performances of the traditional blood transfusion system, for example, problems in blood sample labelling, poor patient identification and mismatched transfusions. Improving the efficiency of blood transfusion, more rapid blood transfusion, reduction in the staff time required for blood transfusion, less wastages, etc. [10] Blood Bag: A Web Application to Manage All Blood Donation and Transfusion Processes: People face many difficulties in getting the suitable or the required blood group at emergencies. Thus, this paper focuses on helping save people's lives by helping them find and locate suitable and reliable blood group that is required by them at the time of emergency. The proposed web based application is connected to the main centralized database, which has the data and records of every blood groups from different blood banks and blood donation campaigns.

\section{Significance of work}

We are helping the patient to get an eligible donor, that is, to check if the donor is fit for blood donation. The donor is first asked for a full health-checkup, i.e., he is checked for contamination of any diseases. Then comes one of the most important criteria to find an eligible donor, on which our project is based. This criteria is checking if the donor has donated previously during that particular year, i.e., the duration between the last blood donation and the current donation should be a minimum of three or more months. Thus, we are using neural network algorithms to train a network that can detect if the donor has donated blood previously during that particular year or not. The area is fruitful for future research since there are many areas which can be improved in the blood transfusion or donation chain. Our project has mainly focused on creating a neural network and then training it to be able to find an eligible donor. One of the area that needs to be addressed in a future research is finding out another criteria for getting an eligible blood donor by using other different algorithms to create and train a neural network. Another area that can be addressed in the future is scheduling of the blood donors. This means keeping a database, consisting of existing and potential donors and using algorithms to schedule the timings of these donors as per the requirements of that particular day.

\section{Methodology}

The data set being used is normalized first and all the values contained in the data set are set in the range [0-1]. Input attributes of the dataset are:-

$\mathrm{R}$ (Recency - months since last time blood was donated)

$\mathrm{F}$ (Frequency - total number of donations by a person)

M (Monetary - amount of blood donated by a person)

$\mathrm{T}$ (Time - months since first time blood was donated)

Of all the values in the dataset, nearly $70 \%$ values are taken as the training set. In our dataset there are around 750 values of blood donors, 500 of those values are used in the training of 
the neural network. Multi-layer perceptron along with back propagation algorithm is used to train the neural network after creating the training dataset. The back propagation algorithm is used, because the neural network must identify if the new input vector is similar to the trained patterns and produces similar kind of results. It is used to observe the local minimum of the error function. The initial weights are then corrected using the gradient of the error function. The algorithm is stopped when the error function becomes small. The neural network is thus created. The remaining $30 \%$ dataset, i.e. around 250 values in our sample is then tested with the already trained neural network.

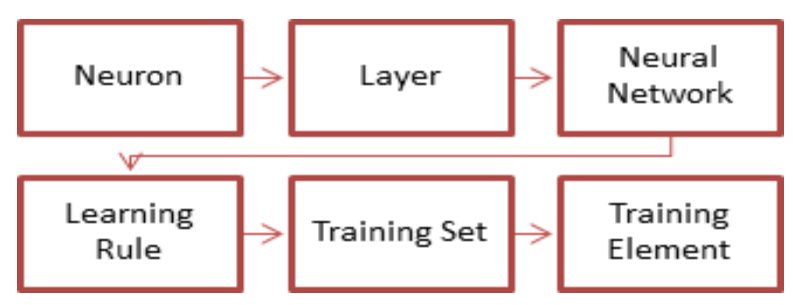

Fig.1. Neural Network Framework and Methodology- Basic Concepts in Neuroph Framework.

Output attributes are:

1 - If a person is eligible to donate blood.

0 - If a person can't donate blood.

\subsection{How blood transfusion can help reduce the energy consumption in medical centers?}

Many blood transfusions that are happening in our country in medical centers consume a lot of energy through the usage of excessive electricity, high voltage machinery, etc. The machines are high voltage devices and require a lot electricity to work efficiently. Through our blood transfusion system, we don't require any additional machinery except the computer. It will be a fully software based methodology which can help in detecting whether a person is eligible to donate blood or not. Only few tests will be conducted and the results will be stored in a database. The computer will parse this data based on the previous training history and predict whether a new patient is eligible to donate blood or not. Many medical centers have set up machines which are costly and require a lot of maintenance. A lot of unnecessary blood transfusions are involved while finding the eligibility of the donor but in our research, we are developing and making the system fully software based with the requirements of least hardware. With energy savings, there is another advantage that the medical centers will get. The costs will significantly reduce thereby increasing the profit and thus they can spend the profits on research or getting world class staff members. We are planning to enhance our system in future by making it work on mobiles, tabs, etc. This will further reduce the consumption of energy that was occurring due to the computers installed in the hospitals. An app will be developed that caters to all the input parameters involved and can use the existing data sets and history to predict the outcome of the blood donor. App will be connected to the hospital server and database and will work as a tool for prediction.

\section{Implementation}

In order to train the neural network, these basic six steps must be performed as shown in 
Figure 1.

Preparation of dataset: For the preparation of the data set, the data set should be normalized first. Normalization means that each values of data set should be in the range 0 to 1 . To achieve this, we used the formula:

$\mathrm{X}_{n}=\left(\mathrm{X}-\mathrm{X}_{\min }\right) /\left(\mathrm{X}_{\max }-\mathrm{X}_{\min }\right)$, where

$\mathrm{X}_{\max }==$ the maximum value of $\mathrm{X}$,

$\mathrm{X}_{\min }=$ the minimum value of $\mathrm{X}$,

$\mathrm{X}_{n}=$ the normalized value of $\mathrm{X}$,

$\mathrm{X}=$ the value of data set which is to be normalized.

Creation of Project: After all the values from the data set are normalized, we need to put these values in a .csv file and then we click on File, and then New Project and a new project is created. After this a new project is created and we are ready to create a new training set.

Then, we create a training set: After we have created a new project, we have to create a training set. We do this by clicking the project and clicking New, and then Training set. Now all the required parameters are filled. Now, we need to minimize the error. So, this is done selecting the type as supervised learning. Supervised learning makes it undergo through a number of iterations and a result, the error is reduced. After this, click on Next and choose the file having the set of values of data set and click on Next.

Then, we create a neural network: After creating the training set, we have to create a neural network. To create a neural network, we right click on the project and click New and then neural network. Now, a window will appear in which we have to select the type of neural network. We have selected the Multi-layer perceptron. This is because it is very easy to use and is robust, that is, it ignores irrelevant inputs, noise or interference. Then, click on Next. Now, here we have to fill the parameters asked. We have checked 'Use Bias Neurons' and chose sigmoid transfer function because the range of our values is between 0 and 1 .

Then, we train the neural network: After we have created the neural network, we have to train the network. To train the neural network, we click on the training set and click on Train. A new window will appear and we set the required parameters.

\section{Results}

After the training of neural network, testing of the data set is performed. The results of testing obtained are as follows:

Table 1. Testing Results.

\begin{tabular}{|c|c|c|c|l|c|}
\hline Input 1 & Input 2 & Input 3 & Input 4 & Output & Error \\
\hline 0.31 & 0.14 & 0.14 & 0.7 & 0.1301 & 0.1301 \\
\hline 0.28 & 0.04 & 0.04 & 0.38 & 0.1301 & 0.1301 \\
\hline 0.31 & 0.04 & 0.04 & 0.34 & 0.1301 & 0.1301 \\
\hline 0.28 & 0.04 & 0.04 & 0.4 & 0.1301 & 0.1301 \\
\hline 0.31 & 0.02 & 0.02 & 0.27 & 0.1301 & 0.1301 \\
\hline 0.28 & 0 & 0.2 & 0.2 & 0.1301 & 0.1301 \\
\hline 0.28 & 0 & 0.2 & 0.2 & 0.1301 & 0.1301 \\
\hline 0.34 & 0.1 & 0.1 & 0.5 & 0.1301 & 0.1301 \\
\hline 0.28 & 0 & 0 & 0.2 & 0.1301 & 0.1301 \\
\hline 0.28 & 0 & 0 & 0.2 & 0.1301 & 0.1301 \\
\hline 0.31 & 0.04 & 0.04 & 0.39 & 0.1301 & 0.1301 \\
\hline 0.28 & 0.02 & 0.02 & 0.32 & 0.1301 & 0.1301 \\
\hline 0.19 & 0.04 & 0.04 & 0.8 & 0.1301 & 0.1301 \\
\hline 0.31 & 0 & 0 & 0.22 & 0.1301 & -0.8699 \\
\hline
\end{tabular}




\begin{tabular}{|l|l|l|l|l|l|}
\hline 0.31 & 0 & 0 & 0.22 & 0.1301 & 0.1301 \\
\hline 0.31 & 0 & 0 & 0.22 & 0.1301 & 0.1301 \\
\hline 0.31 & 0 & 0 & 0.22 & 0.1301 & 0.1301 \\
\hline
\end{tabular}

The network is then tested with several input values chosen from the data set. 5 random values are selected from the dataset.

Table 2. Result Analysis.

\begin{tabular}{|cc|c|c|c|l|}
\hline $\mathrm{R}$ & $\mathrm{F}$ & $\mathrm{M}$ & $\mathrm{T}$ & OUTPUT & ERROR \\
\hline 0.03 & 1 & 1 & 1 & 1 & 0.363940 \\
\hline 0.18 & 0.06 & 0.06 & 0.20 & 0 & 0.378470 \\
\hline 0.05 & 0 & 0 & 0.2 & 0 & 0.379896 \\
\hline 0.03 & 0 & 0 & 0 & 0 & 0.379988 \\
\hline 0.03 & 0 & 0 & 0 & 1 & 0.379988 \\
\hline
\end{tabular}

The last 2 cases have the same inputs but varying outputs. This happened because a majority of people with the same characteristics have not given blood as compared to those who did.

1. Several training and testing iterations were performed on the dataset and the outputs were recorded.

2. The testing results were matched with the results present in the original dataset and after verifying the outputs with the initial dataset, it was noted that the neural network showed an accuracy of $92 \%$.

\section{Conclusion and future work}

We have implemented the blood transfusion system that can help in reducing the energy consumption in medical centers and lead to more profits for the hospitals. Heavy machinery consumes a lot of energy in hospitals and through the use of computers, the dependence on these machines will be less and the hospitals can get the same results with the minimal use of energy. The program will be built using neural networks and use the training data to predict the eligibility of the new donor. Accuracy of the system will depend on the number of training tuples and will be directly proportional to the training data. Only few tests will be conducted and the results will be stored in a database for each donor as a record and will later be used to improve the model by increasing the training data set. The future of the software is bright and it can be implemented in the form of mobile app to further reduce the energy consumptions. Also, the easy to use interface and navigation can help in disseminating the project in hospitals with not so profound knowledge of technology.

\section{References}

1. D.M.S. Kumari, A.N. Wijayanayake, Manufacturing \& Industrial Engineering Symposium (MIES), IEEE Proceedings, (2016)

2. K.K.S. Brailsford, Journal of the Operational Research Society 58, 219, (2007)

3. F. Baronti, A. Lazzeri, R. Roncella, 15th IEEE International Conference on Electronics, Circuits and Systems, (2008) 
4. R.R. Sharma, S. Kumar, S.K. Agnihotri, Vox Sanguinis (2001)

5. B.W.E. Burns, Transfusion, 31, 401 (1991)

6. M. Erraguntla, P. Tomasulo, 47th Hawaii International Conference on System Science, (2014)

7. R. Patil, P. Pawar, M. Poi, Tejashree Patil, Prof. Namrata Ghuse, International Conference on Green Computing and Internet of Things (ICGCIoT), (2015)

8. S.Rani, D. Ganesh, International Journal of Advancements in Research \& Technology, $3,(2014)$

9. S. Pimenta, F. Soares, G. Minas, Conf Proc IEEE Eng Med Biol, (2012).

10. Rehab S. Ali, Tamer F. Hafez, Ali Badawey Ali,Nadia Abd-Alsabour, International Conference on Wireless Communications, Signal Processing and Networking (WiSPNET),(2017) 